\title{
Trans-lingual biopsy of the tongue base under local anaesthetic - a new technique
}

\begin{abstract}
Aim: The tongue base is an important anatomical area to biopsy when searching for an unknown head and neck primary malignancy. Additionally biopsies must be taken when a clinically apparent tongue base lesion is present and malignancy is suspected. It can be extremely challenging to obtain a meaningful biopsy result for a variety of reasons. Patients who present with head and neck malignancy often come with an array of medical co-morbidities and therefore subjecting them to the risks of a general anaesthetic may not be appropriate.
\end{abstract}

Materials and methods: We present a novel technique of tongue base biopsy which can be performed under local anaesthetic. Alongside this we present a case in which this technique has been utilized by the senior author.

Results: The patient presented above had multiple inadequate conventional tonguebase biopsies. Our novel technique yielded a positive result allowing the patient to continue treatment of his recurrent disease.

Conclusion: The trans-lingual biopsy of the tongue base under local anaesthetic is a simple, safe and cost effective way to obtain a tissue diagnosis in tongue base malignancy. We believe it to be reproducible and easy to teach.
Volume 10 Issue 2 - 2018

Jonathan Raihan Abbas,' Joshua Raihan

Abbas, ${ }^{2}$ Paul Hans ${ }^{3}$, John de Carpentier' 'Department of Otolaryngology, Royal Preston Hospital, England

${ }^{2}$ Department of Medicine, University of Glasgow, Scotland ${ }^{3}$ Department of Otolaryngology, Blackpool Victoria Hospital, England

Correspondence: Jonathan Abbas, Department of Otolaryngology, Royal Preston Hospital, Preston, Flat 706 Base Apartments, 12 Arundel st, Manchester, MI5 4JR, England Tel +447584046853,Email jonathan.abbas@doctors.org.uk

Received: December 18, 2017 | Published: March 20, 2018

Keywords: tongue base, tongue, cancer, head and neck, oral, biopsy

\section{Introduction}

Biopsy of the tongue base is an important component of investigating neck metastasis with an unknown primary. ${ }^{1}$ Despite it being a common procedure it is considered to be one of the most difficult areas of the upper aerodigestive tract to access. ${ }^{1-4}$ The difficulty is owing to a variety of factors. The commonest method of accessing the tongue base for biopsy is during a panendocsopy procedure under a general anaesthetic using a pharyngoscope. This is fraught with potential problems due to the need for a general anaesthetic and a high likelihood of false negatives. ${ }^{2}$ We present a novel technique of trans-lingual tongue base biopsy which can be safely performed under local anaesthetic.

\section{Materials and methods}

A 53 year old gentleman re-presented to the otolaryngology department following radiotherapy for a tongue malignancy. He required full work-up for presumed recurrence and therefore was listed for a tongue base biopsy. He underwent this procedure and due to the level of radionecrosis and slough in the tongue base, the biopsy was inconclusive. The patient was therefore referred to the senior author (JDC) for tans-lingual tongue base biopsy. To compound the problem the patient had a complex medical history. He had severe chronic obstructive pulmonary disease (COPD) and had had a previous traumatic brain injury with damage to both cerebral hemispheres. For this reason he was deemed high risk for a general anaesthetic.

The first step of this technique is to ensure adequate infiltration of local anaesthetic. The anaesthetic agent used was LignospanTM (lidocaine $2 \%$ and adrenaline 1:80,000) and was injected using a dental needle and syringe. The anaesthetic was injected in the underside of the tongue, either side of the lingual frenulum. One must inject deep into the muscle of the tongue taking care not to damage the lingual nerve, lingual gland and submandibular ducts. Thorough knowledge of the anatomy of the tongue is vital.

The next step is to make a small incision through the mucosa on the ventral surface of the tongue, again near to the mid-line. The index finger of the non-dominant hand is placed onto the tongue base, just over the lesion or area to be biopsied. The closed biopsy forceps are placed into the mucosal incision and forced through the muscle of the tongue until felt (through the tongue tissue) with the other hand. The mouth of the forceps are opened, pushed into the lesion and then closed and withdrawn. The position of the forceps and the operators non-dominant hand is depicted in Figure 1.

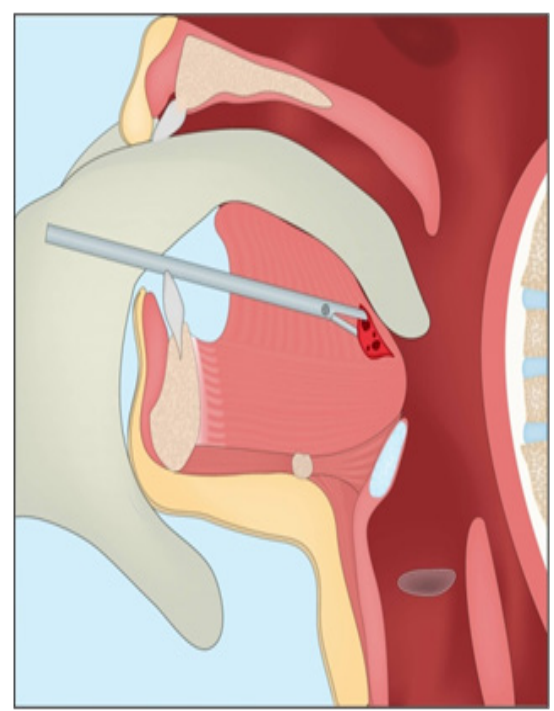

Figure I An illustration of the Local Anaesthetic Trans-Lingual Tongue Base. 
The incision is small and therefore doesn't require formal closure Bleeding is usually minimal due to the surrounding bulk of the tongue musculature. It is of vital importance to maintain a calm operating theatre. Whilst it is generally well tolerated it is invasive and may be daunting for the patient. One must constantly reassure the patient until the procedure is completed.

\section{Results}

The patient described above had, through more conventional methods, previous attempts to gain a tissue diagnosis which failed. Using this novel technique the senior author obtained a substantial deep tongue base biopsy which was diagnostic for neoplastic recurrence. The patient went on to have further treatment to his recurrent disease.

\section{Discussion}

Prompt biopsy of the tongue base is vital for the multidisciplinary team to plan further treatment in patients similar to the one presented above. The procedures can be difficult due to the lack of anatomical access to the area, the fact the malignancy is often submucosal and the potential for overlying slough from previous treatment. ${ }^{3,5}$ In addition, the lesions may often only be palpated and not visualised. ${ }^{3}$ The consequence of these complexities is that samples may be too small and superficial to be diagnostic. ${ }^{2}$

The most conventional method of sampling the tongue base is trans-orally using a pharyngoscope. Blind biopsies may also be undertaken. The often inadequate biopsies have prompted surgeons to attempt to develop new techniques. The trans-lingual biopsy has been previously described by the senior author of this paper but has not been described as being performed under local anaesthetic. ${ }^{3}$

Fine needle aspiration cytology has been trialled in the past with varying results. ${ }^{6-10}$ Pfeiffer et al. Has described the use of transmucosal core needle biopsy of the tongue base. ${ }^{1}$ In a small number of patients it has shown promising results, gaining diagnostic tissue samples. A disadvantage of this technique may be the relative difficulty of aiming the biopsy needle at the suspect area. Other aids to transoral biopsy of the tongue base have been described such as the use of a GlideScopeTM and a video laryngoscope. ${ }^{2,5}$ Whilst certainly interesting they do not tackle the issue of the submucosal nature of the lesion as they only help to visualise the tongue base. The use of robotic surgery in otolaryngology is gaining popularity. Abuzeid et al. described its use in biopsy of the tongue base. This may have more of a role in the future; currently robotic surgery is limited to very few centres globally.

The advantage of this method is that it is a technically simple procedure that relies on the surgeon's ability to accurately advance an instrument towards the finger of their non-dominant hand and has again been shown to provide positive results where other methods have failed.
We perceive the risks to be similar for a conventional tongue base biopsy including; pain, infection, bleeding and need for further surgery due to an incomplete biopsy. As mentioned above care must be taken to ensure the safety of the lingual nerve, lingual gland and submandibular ducts. Our experience is that this method is safe and effective however further research would need to be conducted to accurately quantify possible risks of the procedure.

\section{Conclusion}

We have presented how the trans-lingual tongue base biopsy may be performed under local anaesthetic. We believe that it is a simple procedure which may be considered in medically unfit patients who have failed conventional methods of tongue base biopsy.

\section{Acknowledgement}

None.

\section{Conflict of interest}

None.

\section{References}

1. Pfeiffer J, Maier W, Boedeker CC, et al. Transmucosal core needle biopsy: a novel diagnostic approach to oral and oropharyngeal lesions. $J$ Oral Maxillofac Surg. 2014;72(8):1594-1600.

2. Hughes J, Paul R, O'Flynn P, et al. Use of the Venner TM A.P. Advance TM video laryngoscope for biopsy examination of the base of tongue. $\mathrm{Br}$ J Oral Max Fax surg. 2013;51(2):22-23.

3. Hans PS, Z Rasul, J de Carpentier. A technique for trans-lingual biopsy of the tongue base. Clin Otolaryngol. 2007;32(6):504.

4. Abuzeid WM, Bradford CR, Divi V. Transoral robotic biopsy of the tongue base: A novel paradigm in the evaluation of unknown primary tumours of the head and neck. Head Neck. 2011;35(4):126-130.

5. Shenoy PK, Aldea M. The use of GlideScope for biopsies of the tongue base. JLO. 2013;127(2):215-216.

6. Singh D, Sinba BK, Shyami G, et al. Efficacy of Fine needle aspiration cytology in the diagnosis of oral and oroharyngeal tumors. Intl Arch Otorhinolaryngol. 2008;12(1):99-104.

7. HA Saleh, L Clayman, H Masri. Fine needle aspiration biopsy of intraoral and oropharyngeal mass lesions. Cytojournal. 2008;5(4): 1742-6413.

8. Shah SB, Singer MI, Liberman E, et al. Transmucosal Fine-Needle Aspiration Diagnosis of Intraoral and Intrapharyngeal Lesions. The Laryngoscope. 1999;109(8):1232-1237.

9. Scher RL, Oostingh PE, Levine PA, et al. Role of fine needle aspiration in the diagnosis of lesions of the oral cavity, oropharynx, and nasopharynx. Cancer. 1988;62(12):2602-2606.

10. Buckland JR, Manjaly G, Violaris N. Ultrasound-guided core needle. AJNR Am J Neuroradiol. 2007;69:49. 